\title{
Isolation and information flow in quantum dynamics
}

\author{
Benjamin Schumacher* \\ Kenyon College \\ Michael D. Westmoreland ${ }^{\dagger}$ \\ Denison University
}

\begin{abstract}
From the structure of quantum dynamics for closed and open systems, we derive several general results about information flow between interacting systems, which can be expressed in diagrammatic form. Conditions on information flow (e.g., that no information is transferred from system $A$ to system $B$ ) imply that the overall dynamical evolution has a particular structure. We also show that one simple type of two-qubit interaction, the unitary CNOT gate, cannot be represented by local operations and a single simultaneous information exchange.
\end{abstract}

This paper presents some results about the fbw of information between interacting quantum systems, based on work previously reported by the authors in [1], [2] and [3], together with additional unpublished results. We ask two general questions:

- What does it mean to say that information "fbws" from one quantum system to another during their joint dynamical evolution?

- How is the structure of the dynamical evolution related to the pattern of information fbw between the systems?

In other words, we aim to present some heuristic principles to describe how dynamics and information transfer are related for quantum systems.

Many of our key ideas and results can be represented in a diagrammatic way, based on the quantum circuit model [4]. Time proceeds from left to right in our diagrams, and subsystems are represented by horizontal lines.

- The input end of a line may either be free or fixed. A fixed input (represented by the $\diamond$ symbol) is specified to be prepared in some fixed standard state $|0\rangle$ that is uncorrelated with the rest of the system. The joint state of the free inputs may be any product or entangled state.

- At the output end, some subsystems may be discarded (represented by the symbol $\otimes)$. We only consider the output state of the non-discarded subsystems.

- The interactions among systems are shown by boxes. These interactions may either be unitary (white boxes) or a more general, open-system evolution (gray boxes).

*schumacherb@kenyon.edu

†westmoreland@denison.edu 
To illustrate these conventions, we recall that any evolution map $\mathcal{E}$ on density operators of a system $A$ must be a trace-preserving, completely positive map. Such a map can always be represented by unitary evolution on a larger system $A E$, where the "environment" system $E$ is initially in a given state $|0\rangle$. As an equation, this means that for any initial $A$-state with density operator $\rho$,

$$
\mathcal{E}(\rho)=\operatorname{Tr}_{E} U(\rho \otimes|0\rangle\langle 0|) U^{\dagger} .
$$

This fact has a diagrammatic representation like this:

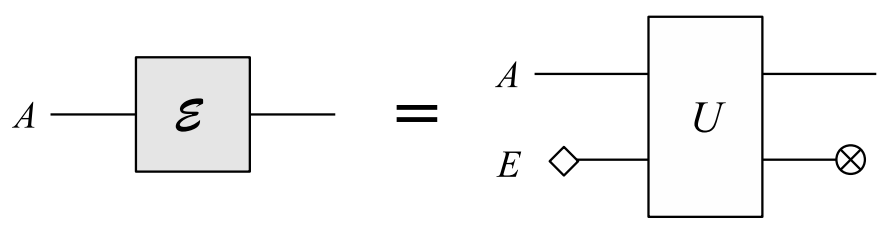

A unitary interaction has the same input and output systems. However, a more general map could relate input states and output states for systems of different size. For example, an interaction of two systems for which one input is fixed is effectively a "one-input, twooutput" interaction. An interaction of two systems in which one output is discarded is effectively a "two-input, one-output" interaction.
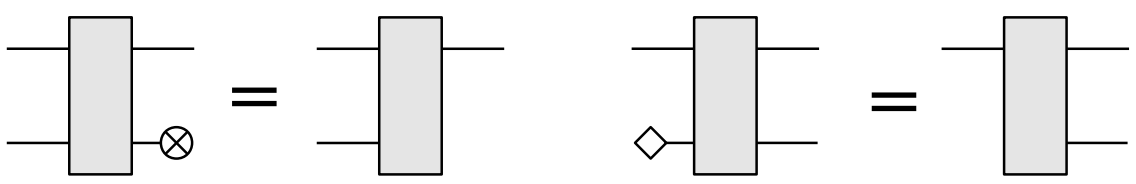

We define what we mean by "information fbw" by defining its opposite. Suppose a system is composed of subsystems including $A$ and $B$ and possibly others (collectively denoted $X$ ). The entire system $A X B$ evolves according to some general evolution map $\mathcal{E}$. We say that information does not fbw from $A$ to $B$ (a condition we denote $A \leadsto B$ ) provided we can determine the final state of $B$ from the initial state of $X B$ alone. That is, $A \uparrow \rightarrow B$ means that we can eliminate the $A$ input in the diagram below:
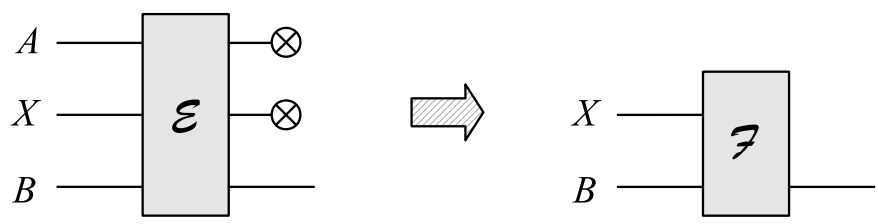

This condition implies that no prior operation on subsystem $A$ can lead to any observable effect on the output state of $B$, regardless of the joint initial state of $A X B$. Thus, the evolution of $A X B$ cannot be used to send a message from $A$ to $B$.

The isolation theorem. Our first result may be termed the isolation theorem. We imagine two subsystems $A$ and $E$, with $E$ in a fixed initial state. The composite system evolves in a unitary way according to some operator $U$. If we discard $E$ at the end, we end up with a general evolution map $\mathcal{E}$ for system $A$ alone. The isolation theorem states that this map is in fact unitary evolution (for some $A$-operator $V$ ) if and only if $A \leadsto E$.

As a diagram, we can say that the condition $A \leadsto t E$ implies that we can make the following reduction: 


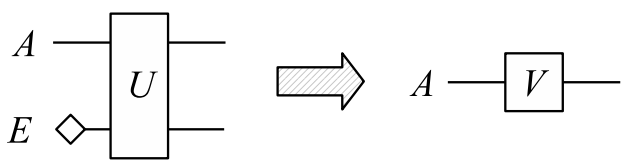

The theorem further says that the possibility of this reduction implies $A \leadsto t E$.

A quantum system evolves in a unitary way if and only if none of its information "leaks" into the environment- the system is informationally isolated. This simple fact has many applications. In the well-known two-slit experiment, for instance, the interference effects are due to the linear (unitary) evolution of the wave function of the quantum particle passing through the barrier. These effects appear only if the passage of the particle through the barrier leaves no physical record anywhere of which slit the particle goes through.

The isolation theorem also provides a simple proof of a strong form of the quantum no-cloning theorem. An ideal cloning machine would leave the input state of the original system unchanged - that is, the state $|\psi\rangle$ of the original system evolves according to the unitary operator 1 . But this can happen only if the final environment state-including any "clone" systems—is completely independent of $|\psi\rangle$.

Locality in quantum evolution. We now turn to a collection of results designed to shed light on the idea of "local evolution" in quantum theory. We say that the evolution of a system is local if the future state of a subsystem $B$ will depend on the initial state of $B$ and its immediate surroundings (subsystem $X$ ), but not on the more distant surroundings, represented by subsystem $A$. In other words, $A \ngtr \rightarrow B$ during the period of time under consideration.

Suppose that the overall evolution of the system $A X B$ is unitary, given by an operator $U^{(A X B)}$. Then we can prove that $U^{(A X B)}$ may be decomposed as follows:

$$
U^{(A X B)}=\left(W^{(A X)} \otimes 1^{(B)}\right)\left(1^{(A)} \otimes V^{(X B)}\right) .
$$

As a diagram, this means that

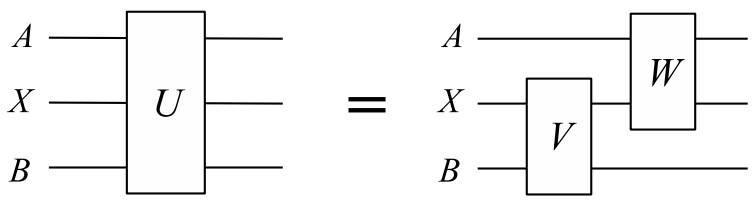

Clearly, any unitary interaction of this form must have the property that $A \leadsto B$, since the final state of $B$ is already determined before $A$ interacts with $X$.

What about more general maps for $A X B$ ? In the general case, $A \leadsto B$ does not imply that the overall dynamics may be into an $X B$ interaction followed by an $A X$ interaction. However, if $A \leadsto B$ then the overall dynamics must have a unitary representation of the form

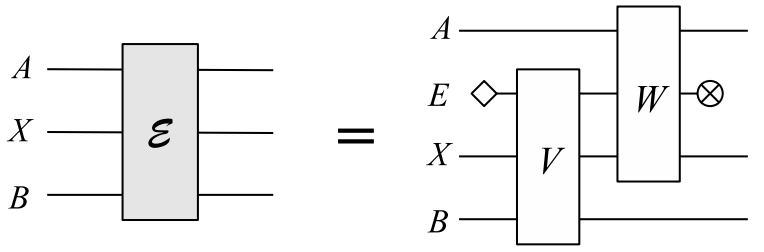


for some environment system $E$.

Decomposing CNOT. One of the most elementary two-qubit gates in the quantum circuit model is the "controlled-NOT" or CNOT gate. It is usually taken to be one of the basic building-blocks for constructing more complex quantum information processes. Here we investigate whether this simple interaction can itself be decomposed into simpler pieces.

Any classical two-input, two-output computation can be performed by local computations and a single, simultaneous exchange of information. If Alice and Bob each possess one input of the computation, and they must each obtain one output, they can accomplish this by simply sending each other copies of their inputs and independently doing the full computation. However, it is not possible to copy quantum information, so this simple procedure is not available. Can a two-input, two-output quantum computation such as CNOT be done by Alice and Bob using simultaneous information exchanges?

It turns out that the CNOT cannot be done in this way. That is,
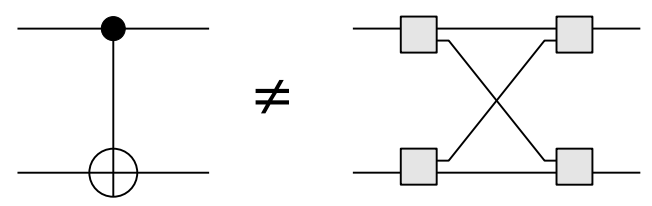

for any possible choice of four independent dynamical evolutions in the right-hand diagram. The CNOT can be done in other ways-by successive information exchanges, and (somewhat less trivially) by making use of prior shared entanglement. This result shows that a simple, simultaneous exchange of information is not an adequate picture of the information fbw in the CNOT gate.

Our result for CNOT has been generalized by Nathanson to a more general class of unitary interactions between qubits [5].

\section{References}

[1] B. Schumacher and M. D. Westmoreland, "Locality and information transfer in quantum operations," Quantum Information Processing 4, 13 (2004).

[2] B. Schumacher and M. D. Westmoreland, Quantum Processes, Systems and Information (Cambridge University Press, Cambridge, 2010).

[3] B. Schumacher, "Interaction and information fbw between quantum systems (Part 1B)", talk at the 2007 Quantum Foundations Summer School at the Perimeter Institute (http://pirsa.org/06070007/).

[4] M. A. Nielsen and I. L. Chuang, Quantum Computation and Quantum Information (Cambridge University Press, Cambridge, 2000).

[5] M. Nathanson, private communication. 